Rafidain Journal of Science

Vol. 29, No.2, pp. 51-63, 2020

DOI

https://rsci.mosuljournals.com

ISSN: 1608-9391 e-ISSN: 2664-2786

Received $11 / 6 / 2019$

Accepted 26/11/ 2019
تأثير التدعيم بمساحيق الألومينا وإلسليكا على الذصائص الميكانيكية للخليط البوليمري (بولي أستر غير مشبع/بولي يوريثان)



*E-mail: dbqpman@yahoo.com dr.raadrasool@yahoo.com** seham_yns@yahoo.com***



نم في هذه الدراسة تحضير مواد متراكبة ذات أساس من الخليط البوليمري (B) (UPE/PU, 95/5) (150 رm - 75). بعدها نم اختبار النماذج المحضرة وهي الخليط البوليمري (B) والمواد


المتمثلة باختبار الانحناء (Bending Test) والإنضغاط (Compression) والصلادة والصدمة (Hardness) بأن أكبر قيمة لمعامل يونك من اختبار الانحناء كانت 6.15 GPa في العينة B. مقاومة الكسر قلت مع زيادة التدعيم. أما بالنسبة لمقاومة الإنضغاط فإن أقصى مقاومة


الصلادة فقد ازدادت مع زيادة التدعيم حيث بلغت أقصى قيم لهما 94.5 Hs. الكلمات الدالة: مواد متراكبة، خليط بوليمري، خصائص ميكانيكية. 


\section{المقدمة}

إن للبوليمرات أهمية كبيرة في حياة الإنسان حيث أن معظم الصناعات الحالية قائمة على هذه المركبات الكيميائية، ولقد


تمند من أدوات محلية شائعة إلى أجهزة علمية وطبية منطورة (Crawford, 1998). ومن مميزات المواد اللدائنية هي التكلفة




الحراري (الرفاعي، 2011)، على الرغم من هذه المميزات وغيرها الكثير إلا أن البوليمرات بشكلها الخام تفشل في أحيان كثيرة في


أخرى تعرف بالحاشيات (Crawford, 1998)، وبهذا يستحصل منها على مواد منراكبة وهي عبارة عن جمع مادنين أو أكثر




(Fiber Glass من ليف إلى آخر وبذلك تثقاسم الألياف الحمل المسلط (Battelle, 2008). وتمتاز الخصائص الميكانيكية للمتراكبات المعززة بالدقائق عن تللك المعززة بالألياف بكونها بصورة عامة متتاظرة، حيث أنه لا يوجد تغيير في الخصائص الميكانيكية بتغيير الاتجاه


مما يقلل من كلفة استخدامها اقتصاديا(محمد، بوليمرات أخرى مشكلة خلائط بوليمرية (Polymer Blend) ويستفاد من هذه الطريقة كذلك في تقليل الكلفة وهي أسهل من انتاج


بتحضير مواد متراكبة بوليمرية مقواة بمواد تقوية مختلفة حيث أجرى (العزاوي وآخرون، 2010) دراسة لتأثنير درجة الحرارة والمحاليل الكيميائية على قيم معامل المرونة لمواد منراكبة هجينة مكونة من خليط بوليمري من راتتجات الفينول فورمالديهايد نوع دواهي نوفولاك والإيبوكسي المقوى بمساحيق الألومينا والسليكا بالإضافة إلى ألياف الإسبستوس وبكسور حجمية (30\%, 40\%)، ونم تحضير ستة مواد متراكبة هجينة وإجراء إختبار الانحناء لحساب معامل المرونة في درجات حرارية مختلفة ومحاليل كيميائية مختلفة. أظهرت النتائج أنه كلما ارتفعت درجة الحرارة قلت قيم معامل المرونة، كذلك كلما ازدادت فنرة غمر العينات في المحاليل الكيميائية المختلفة قلت قيم معامل المرونة، وأظهرت نتائج اختبار العينات التي تحتوي على خليط من الألومينا والسليكا وبكسر حجمي 40\% زيادة في قيم معامل المرونة في حين أن العينات التي تحتوي على الألومينا بكسر حجمي 30\% أظهرت أقلى أقل قيم






لها هي عند النسبة الوزنية (10\%) وكانت 229 و 180 وبوحدة MPa للألومينا والتيتانيوم على التوالي. أما الصلادة فإن قيمتها تزداد بزيادة نسبة التدعيم وكانت أعلى قيمة عند النسبة (15\%) وبقيمة 15 و 12 وبوحدة BNH لكل من النماذج المقواة بدقائق


حجمية مختلفة (10\%, 20\%) إلى خليط من (UPE/PU) وبنسب وزنية (10/10). أظهرت النتائج أن إضافة الدقائق إلى الخليط البوليمري قد حسن وبشكل كبير الخواص الميكانيكية المتمنلة بالانحناء والصلادة والصدمة ومعامل المرونة. 
يهدف البحث إلى تحضير مواد متراكبة ذات أساس من خليط بوليمري مدعم بمساحيق الألممينا والسليكا وبكسور حجمية


الصدمة) والاستفادة منها في الصناعة.



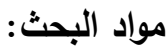

راتنج البولي أستر غير المشبع (Unsaturated Polyester (UPE)

تم استخدام راتتج البولي أستر غير المشبع نوع (TP 100) المنتج من الثركة التركية (Turkuas Polyester)


يتحول من الحالة السائلة إلى الحالة الصلبة بإضافة المصلا (Hardener) له والمصلد عبارة عن مثيل أثنيل كيتون بيروكسايد (25/75, (اسيتون/مصلد) (Methyl Ethyl Keton Peroxide, MEKP) واستعمال (0.03g) من هذا الناتج لكل (10g) من راتتج البولي أستر بهدف التصليد، وذلك بسبب صعوبة استخدام المصلد لوحده بدون الأسيتون بسبب سرعة التصلد العالية والتي تستغرق ثلاث دقائق. الجدول (1) يوضح بعض من خصائص البولي أستر غير



الجدول 1: بعض من خصائص البولي أستر غير المشبع

\begin{tabular}{|c|c|c|c|c|c|}
\hline $\begin{array}{c}\text { Product } \\
\text { Name }\end{array}$ & $\begin{array}{c}\text { Density } \\
\left(\mathbf{g m} / \mathbf{c m}^{\mathbf{3}}\right)\end{array}$ & $\begin{array}{c}\text { Flexural } \\
\text { Strength } \\
(\mathbf{M P a})\end{array}$ & $\begin{array}{c}\text { Flexural } \\
\text { modulus } \\
\mathbf{( G P a )}\end{array}$ & $\begin{array}{c}\text { Compressive } \\
\text { Strength } \\
(\mathbf{M P a})\end{array}$ & $\begin{array}{c}\text { Fracture } \\
\text { toughness } \\
\mathbf{M P a} \sqrt{\mathbf{m}}\end{array}$ \\
\hline UPE & $(1.1-1.5)$ & $59-159$ & $3-4.2$ & $90-250$ & 0.6 \\
\hline
\end{tabular}

بولي يوريثان Poly Urethane (PU):


تحضيره من خلط البوليمر مع المصلد (آيزوسيانيد) بنسبة جزأين من البوليمر إلى جزء واحد من المصليد.

مسحوق السليكا SiO

نم استخدام مسحوق السليكا بحجم حبيبي يتراوح بين Mm (150-75) والذي تم تحديده باستخدام المناخل، والمسحوق منتج من قبل شركة (Filcom a Sibelco Company) وهو مسحوق أبيض اللون وعديم الذوبان في الماء. و (الجدول 2) يوضح بـاني بعض من الخصائص السليكا والألومينا (Callister, 2009)، (Coble, 2003)، (Doremus and Shackeford, 2008). 


$$
\text { حنين محسن علي واخرون }
$$

الجدول 2: بعض خصائص السليكا والألومينا

\begin{tabular}{|c|c|c|c|c|c|c|}
\hline $\begin{array}{c}\text { Product } \\
\text { Name }\end{array}$ & $\begin{array}{c}\text { Density } \\
\left(\mathbf{g m} / \mathbf{c m}^{\mathbf{3}}\right)\end{array}$ & $\begin{array}{c}\text { Knoop } \\
\text { hardness } \\
\mathbf{( 1 0 0} \mathbf{g m} \\
\mathbf{L o a d})\end{array}$ & $\begin{array}{c}\text { Young's } \\
\text { modulus } \\
(\mathbf{G P a})\end{array}$ & $\begin{array}{c}\text { Compressive } \\
\text { Strength } \\
\mathbf{( M P a})\end{array}$ & $\begin{array}{c}\text { Flexural } \\
\text { Strength } \\
\mathbf{( M P a})\end{array}$ & $\begin{array}{c}\text { Fracture } \\
\text { toughness } \\
\mathbf{M P a} \sqrt{\mathbf{m}}\end{array}$ \\
\hline $\mathbf{S i O}_{\mathbf{2}}$ & 2.65 & 800 & 83 & $650-1100$ & 100 & 0.79 \\
\hline $\mathbf{A l}_{\mathbf{2}} \mathbf{O}_{\mathbf{3}}$ & $\begin{array}{c}3.97- \\
3.986\end{array}$ & 2100 & 390 & $2070-2620$ & $282-551$ & $4.2-5.9$ \\
\hline
\end{tabular}

مسحوق الألومينا Aluminum Oxide $\left(\mathrm{AL}_{2} \mathrm{O}_{3}\right.$ : تم استخدام مسحوق ألومينا بحجم حبيبي يتراوح بين (Am بm (75-150) والذي نم تحديده باستخدام المناخل، وهو من المساحيق

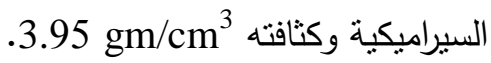
تحضير نماذج البحث:


مدعمة بنسب حجمية مختلفة من مساحيق الألومينا والسليكا المايكروية بطريقة القولبة اليدوية و (الجدول 3) يوضح نسب مكونات




(20*10*10)، (55*10*10) لاختبارات الانحناء والانضغاط والصدمة على التوالي ونم إجراء معاملة حرارية للعينات عند 50 (10)

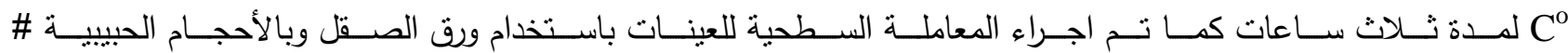
.$(100,200,400,600,800,1000)$

الجدول 3: رموز العينات المحضرة والنسب الحجمية للمواد الداخلة في تحضيرها

\begin{tabular}{|c|c|}
\hline $\begin{array}{c}\text { Symbols of the } \\
\text { Samples }\end{array}$ & $\begin{array}{c}\text { Percentage of each Material in the } \\
\text { Samples }\end{array}$ \\
\hline $\mathbf{B}$ & Blend (UPE 95\%+PU 5\%) \\
\hline $\mathbf{B}_{\mathbf{1}}$ & Blend $95 \%+\mathrm{Al}_{2} \mathrm{O}_{3} 5 \%$ \\
\hline $\mathbf{B}_{\mathbf{2}}$ & Blend $80 \%+\mathrm{Al}_{2} \mathrm{O}_{3} 5 \%+\mathrm{SiO}_{2} 15 \%$ \\
\hline $\mathbf{B}_{\mathbf{3}}$ & Blend $65 \%+\mathrm{Al}_{2} \mathrm{O}_{3} 5 \%+\mathrm{SiO}_{2} 30 \%$ \\
\hline
\end{tabular}
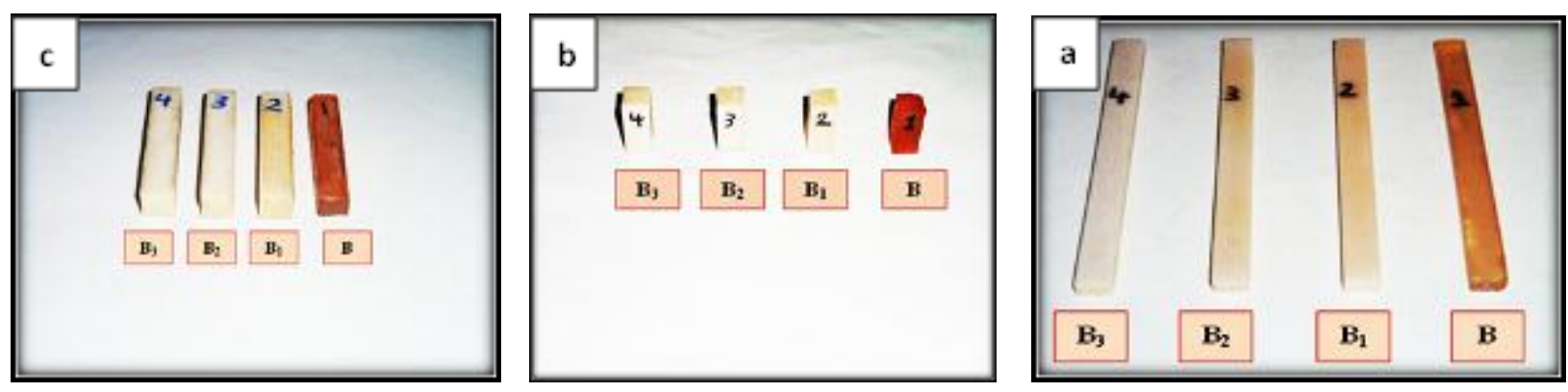

الشكل 1: صور لنماذج العينات المحضرة لاختبارات (a) الانحناء، (b) الانضغاط، (c)(الصدمة 


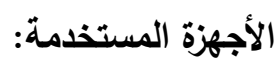

نم استخدام جهاز (SANS, Model WAW-1000) صيني المنشأ في جامعة الموصل/ كلية الهندسة الميكانيكية/مختبر المعادن، لإجراء إختبارات الإنحناء والإنضغاط. وتم إستخدام جهاز (Shore Durometer, 0-100 HD) صيني المنشأ لغرض إجراء إختبار الصلادة كما تم الحصول على الطاقة الممتصة اللازمة لحدوث الكسر في العينات لجهاز قباس طاقة الصدمة جاربي (Charpy Impact Instrument) المجهز من شركة ( Tokyo Koki Seizosho, LTD).

\section{النتائج والمناقشة}

Bending Test إختبار الإنحناء

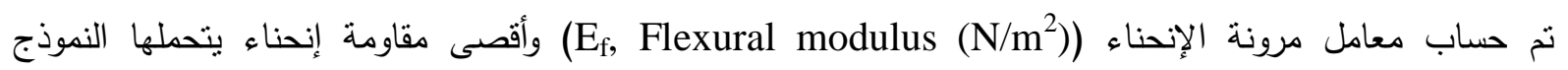
$\left(F_{S B}\right.$, Flexural Strength at Break $\left(\mathrm{N} / \mathrm{m}^{2}\right)$ ) ومقاومة الكسر ( $\mathrm{F}_{\mathrm{SM}}$, Maximum Flexural Strength $\left.\left(\mathrm{N} / \mathrm{m}^{2}\right)\right)$ للنماذج المختبرية باستخدام العلاقات التالية (Ratna, 2009)،(Akay, 2012):

$$
E_{f}=\frac{L^{3}}{4 b h^{3}} \frac{P}{S}
$$

حيث إن:

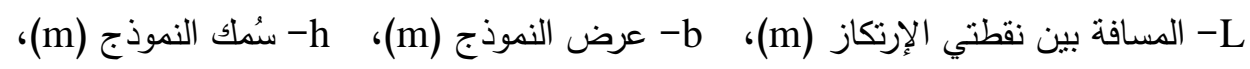

$F_{S M}=\frac{3 P_{M} L}{2 b h^{2}}$ يمنل ميل الجزء الخطي للعلاقة بين الحمل P والإنحراف S بوحدة (N/m).

$F_{S B}=\frac{3 P_{B} L}{2 b h^{2}}$ حيث إن :

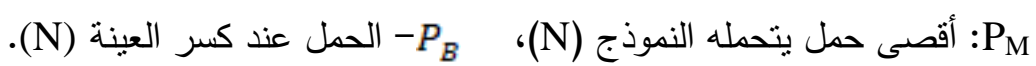

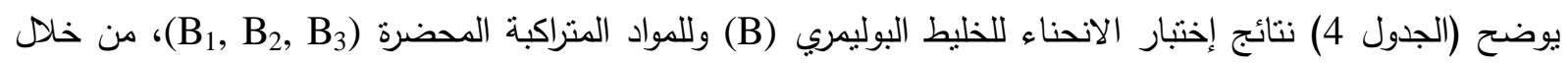

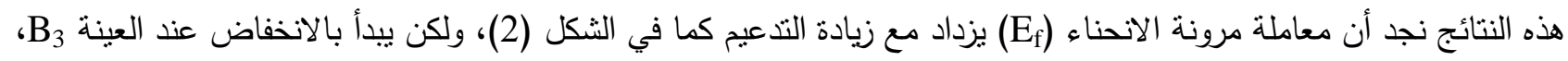

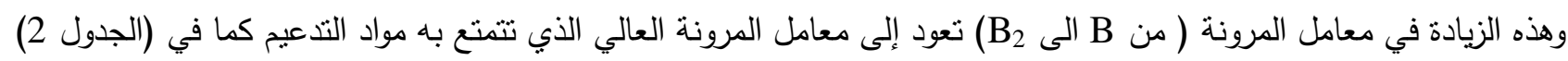

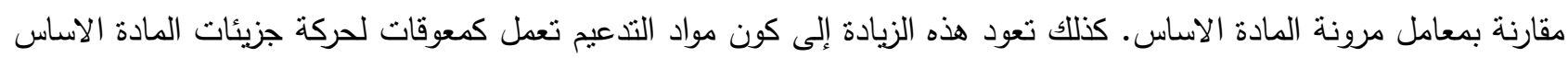

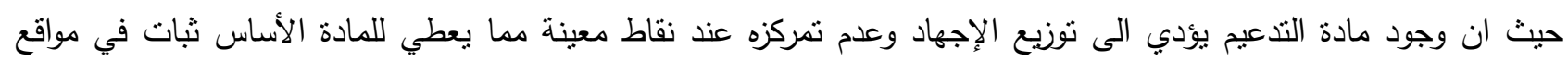
جزيئاتها والذي سوف ينعكس على قلة الانفعال المرن مع زيادة مواد التدعيم وكما مبين في الثكل رقم

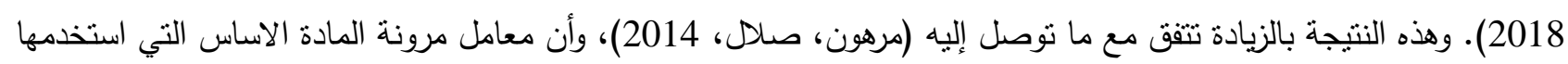
الباحث مقارب لـ (2.7 GPa)، وقد يعود هذا الفرق لوجود البولي يوريثان ضمن المادة الأساس في بحثنا الحالي. 
الجدول 4: نتائج إختبار الإنحناء للعينات المحضرة

\begin{tabular}{|c|c|c|c|}
\hline رمز العينة & $\mathbf{E}_{\mathbf{f}}(\mathbf{G P a})$ & $\mathbf{F}_{\mathbf{S M}}(\mathbf{M P a})$ & $\mathbf{F}_{\mathbf{S B}}(\mathbf{M P a})$ \\
\hline $\mathbf{B}$ & 1.15 & $32.67^{*}$ & ------- \\
\hline $\mathbf{B}_{\mathbf{1}}$ & 2.56 & 39.03 & 39.03 \\
\hline $\mathbf{B}_{\mathbf{2}}$ & 6.15 & 30.08 & 29.87 \\
\hline $\mathbf{B}_{\mathbf{3}}$ & 4.19 & 25.56 & 25.56 \\
\hline
\end{tabular}

"تم حسابها عند الإنفعال 5\% لأنها لم تتكسر بعد هذه القيمة من الإنفعال وذلك حسب مواصفات (ASTM-D790)

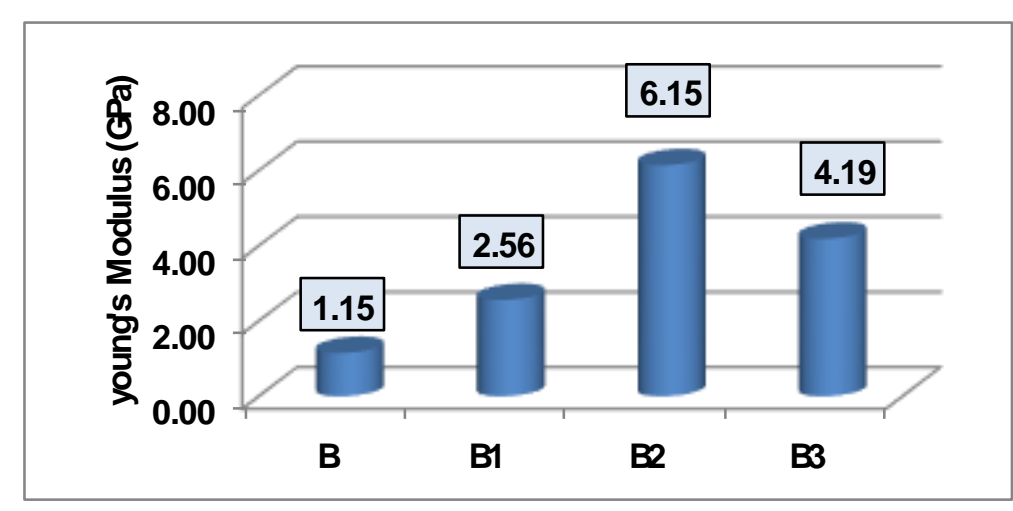

الشكل 2: معامل مرونة الانحناء لنماذج العينات.

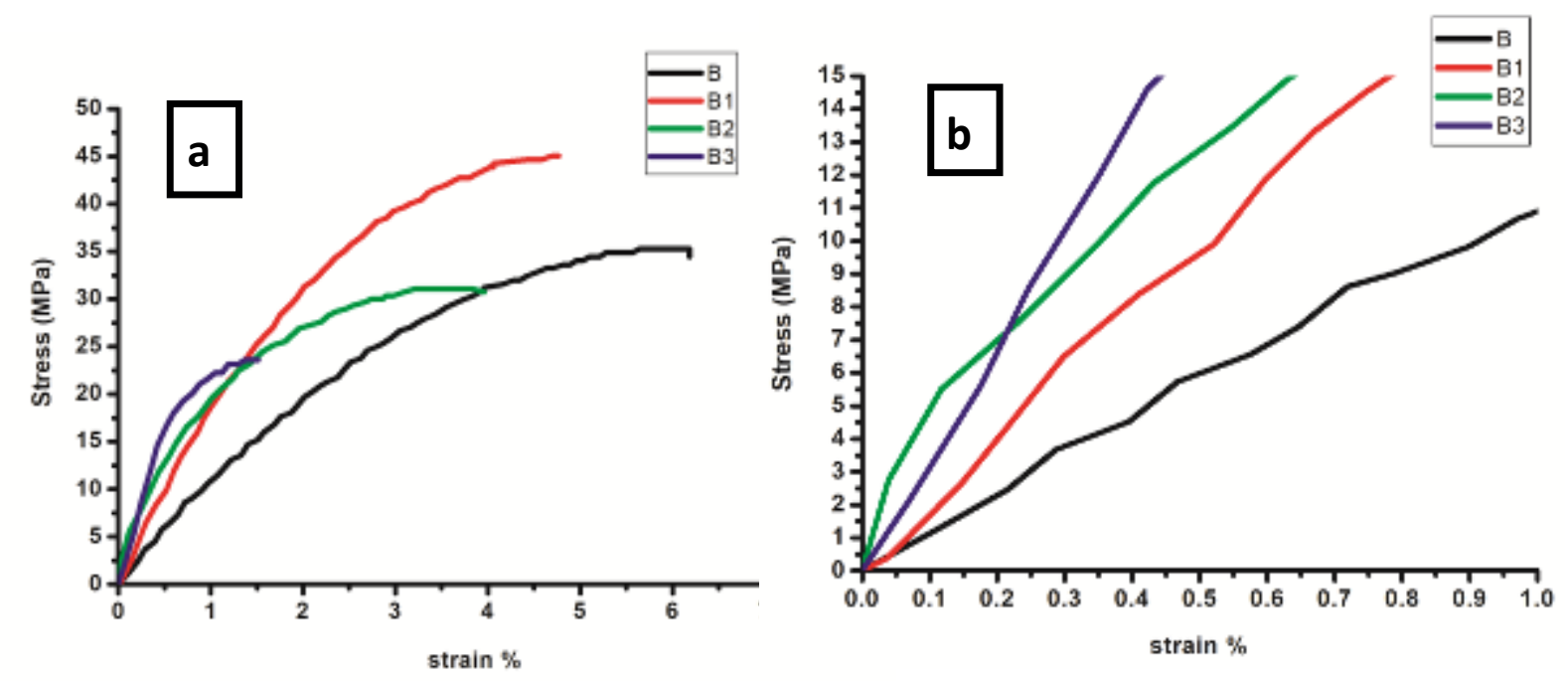

الثكل 3: (a) منحنيات الاجهاد-الانفعال للعينات، (b) تكبير لمنطقة تحديد معامل المرونة

وتتفق هذه النتائج كذلك مع ما توصلت إليه (Musa, 2014) حيث لم تتجاوز نسب التدعيم الحجمية التي استخدنها الباحثة vol\% 20 وكانت أعلى قيمة لمعامل مرونة الانحناء عند هذه النسبة من التدعيم، كما أن المادة الأساس التي استخدمتها 
الباحثة هي (UPE/PU, 90/10)، ومعامل المرونة لها في حدود (0.7 GPa) وهي أقل من معامل مرونة المادة الأساس في

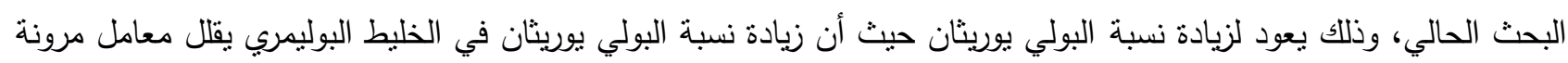

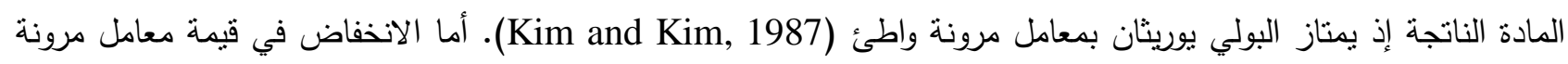
الإنحناء عند العينة B33 يعود لزيادة نسبة التدعيم الى المادة الأساس مما يؤدي إلى أضعاف الى ارتباط مادة التدعيم مع المادة

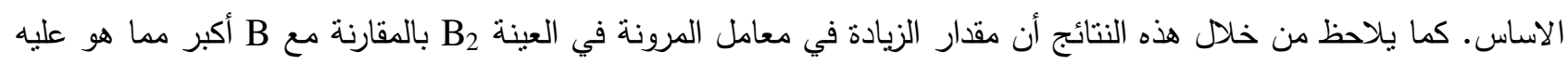

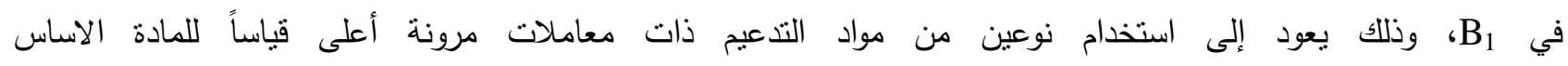



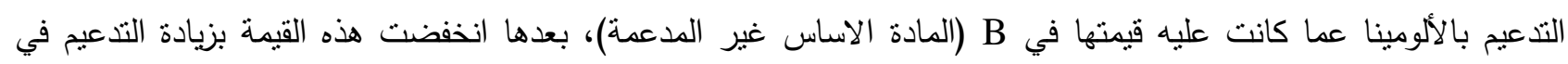

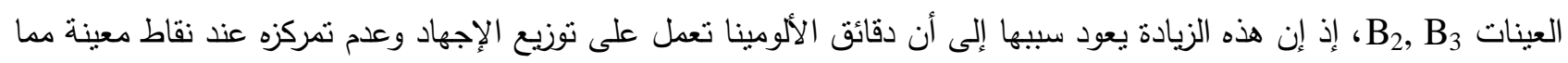

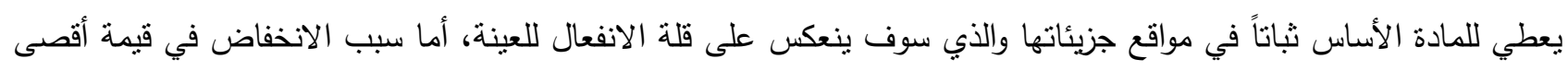

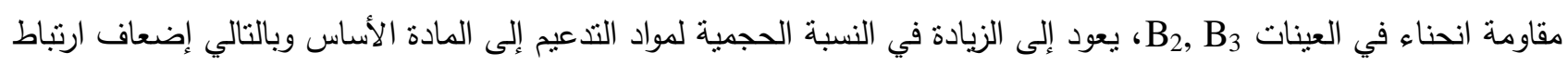
مادة التدعيم مع المادة الاساس وتكتل مواد التدعيم (Hanna et al., 2011 ) هذه النتيجة تتفق مع ما توصل إنى إليه (Durowaye, 2018)، إذ حصلوا على أعلى مقاومة إنحناء وكانت عند نسبة التدعيم vol\% 7 voادة وباستخدام مسحوق كربيد السليكون حيث عند زيادتهم لنسبة التذعيم حصلوا على انخفاض في مقاومة الانحناء.

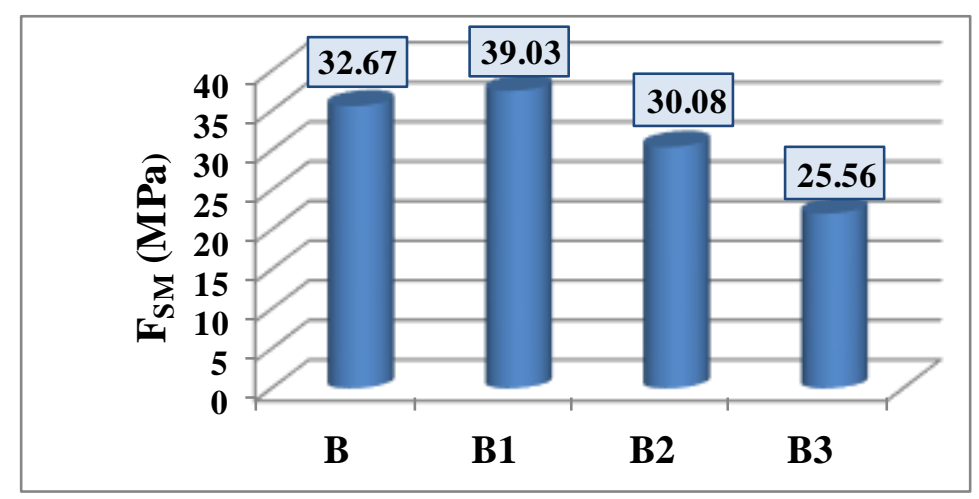

الشكل 4: أقصى مقاومة اتحناء تتحملها النماذج.

كما يلاحظ من خلال نتائج الإختبار الموضحة في (الجدول 4) لمقاومة الكسر للعينات المحضرة وكما في الثكل (5)، يلاحظ عدم كسر العينة B1 وذلك لخصائص في لدونة المادة الأساس وتأثير مطاط البولي يوريثان، إذ تمتاز البوليمرات المطاطية

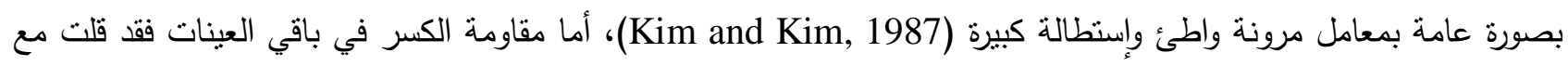

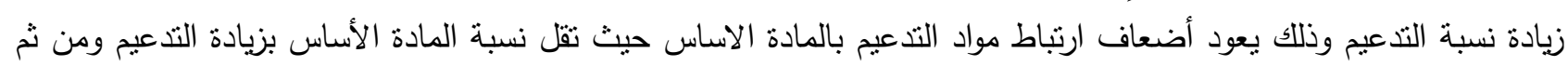

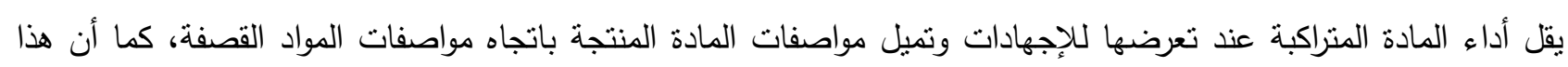
الإنخفاض يعود إلى إنخفاض نسبة المادة الاساس، حيث أن نسبتها الحجمية تقل مع زيادة نسبة مواد التدعيم وتفقد المادة المتراكبة نتيجة لللك اللدونة المستمدة من خصائص المادة الاساس (Rodriguez, 2015). 


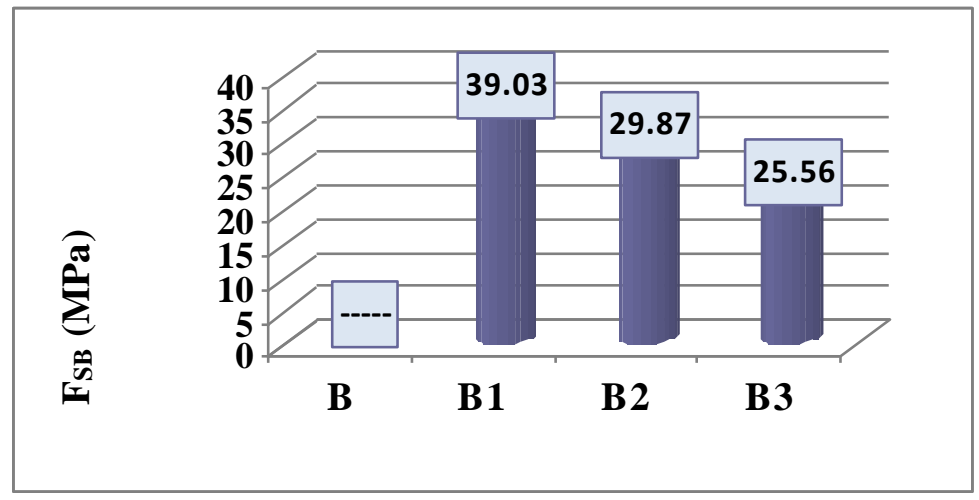

الشكل 5: مقاومة الكسر لنماذج العينات

Compression Test إختبار الإنضغاط

يعد اختبار الانضغاط حالة عكسية لاختبار الثد من حيث اتجاه الحمل المؤثر كما يمنل اختبار الثد الأساس لقبول المواد المعدنية فإن اختبار الانضغاط الأساس لقبول المواد غير المعدنية مثل الخرسانة والطوب والأحجار والأخشاب، إذ إنها تكون

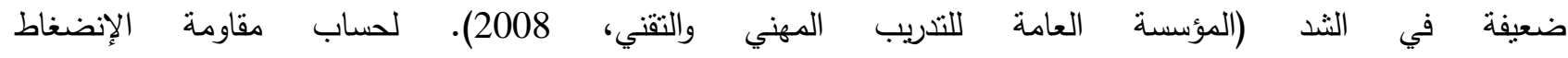
(Callister, 2009) نم الاعتماد على العلاقة الآتية (Compressive Strength, C.S)

$$
\text { C.S }(P a)=\frac{\text { Force }(N)}{\text { Area }\left(m^{2}\right)}
$$

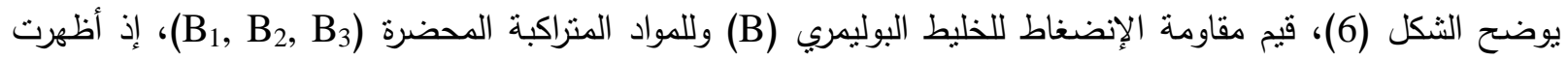

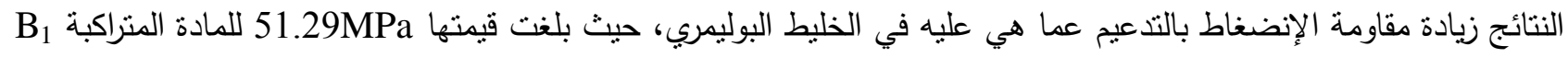

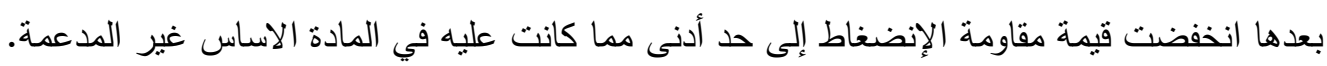

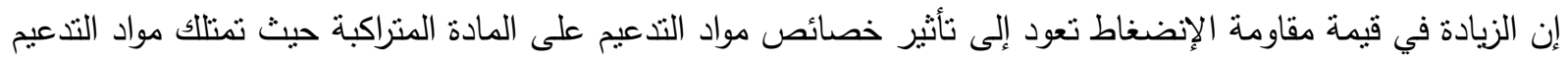
الألومينا والسليكا مقاومة انضغاط عالية وكما موضح في (الجدول 2)، بالمقارنة مع المادة الأساس، وتؤدي عملية التدعيم إلى مزج مونج هذه الصفات النوعية لمواد التدعيم مع صفات المادة الأساس (تاكر، 1984)، كما أن مواد التدعيم هي مواد سيراميكية وتعد مواداً مسامية (مرهون، صلال، 2014)، إذ نوفر هذه الدقائق سطح إمتزاز جيد (Absorbent) لجزيئات البوليمر مما يؤدي إلى زيادة

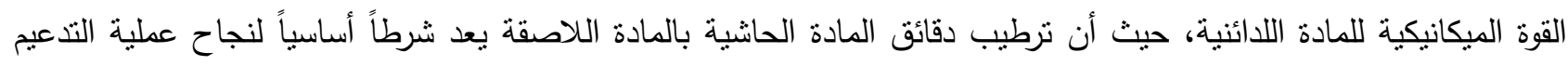
(تاكر، 1984). ومن مقارنة النتائج يلاحظ أن العينة B أظهرت أعلى قيمة لمقاومة الانضغاط بسبب وجود الألومينا ذات مقاومة

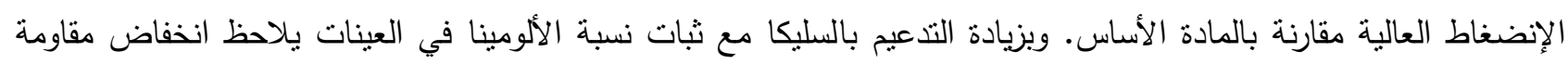

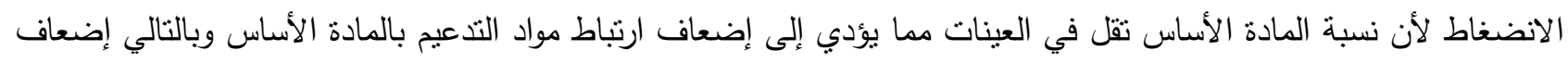




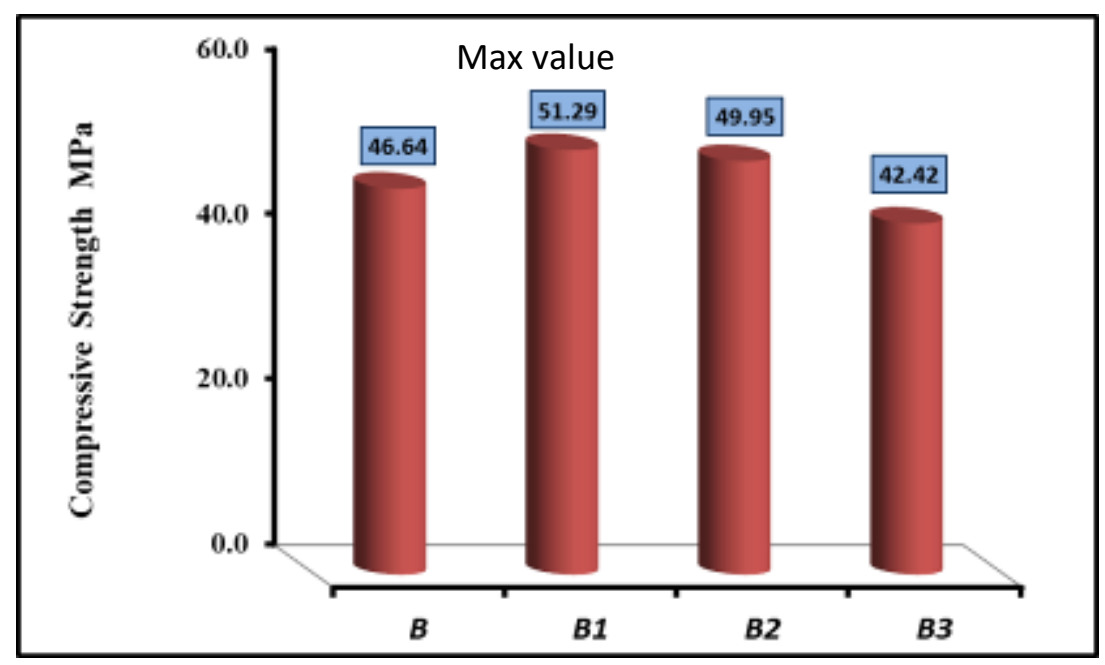

الشكل 6: نتائج إختبار تغير مقاومة الإنضغاط للنماذج المحضرة

Hardness Test إختبار الصلادة

إن الهدف من اختبار الصـلادة هو قياس مقاومـة المادة للتشوهات اللدنة (حسين، حسين، 2010)، والثكل (7) يوضـح

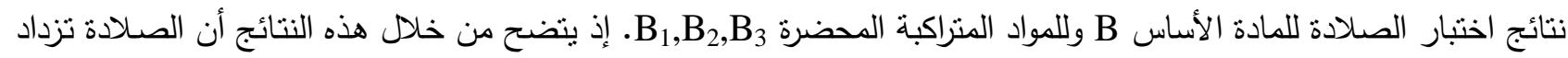
بزيادة التدعيم بالمقارنة مع المادة الأساس B. يعود هذا إلى تأثثر خصائص الصـلادة العالية لمواد التدعيم كما في (الجدول 2) بالمقارنـة مـع المادة الاسـاس، إذ تضاف هذه الصفة للمادة المتراكبة عند التدعيم (حسن، 2017) حيث ان وجود المواد المدعمة بخصائصـها يؤثر بعاملين، الأول: زيادة في تماسك المادة الأسـاس والثاني: تطوير خواص المـادة الأسـاس بسبب الاقتران بين خصائص المادة الأساس ومادة التدعيم. كما أن وجود مواد التدعيم (الألومينا والسليكا) بين جزيئات المادة الأساس سيؤدي إلى لى إعاقة حركة هذه الجزيئات مما أدى لزيادة صعوبة إحداث أثز على السطح في عينات المواد المتراكبة وبالتالي سيعمل على زيادة

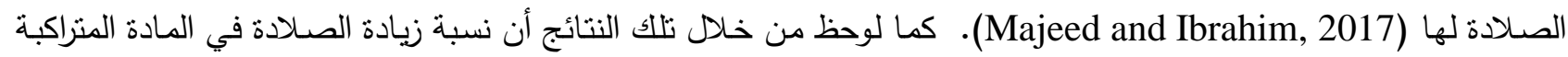

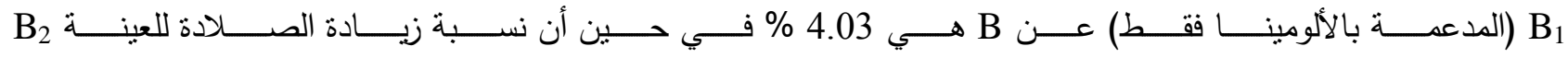

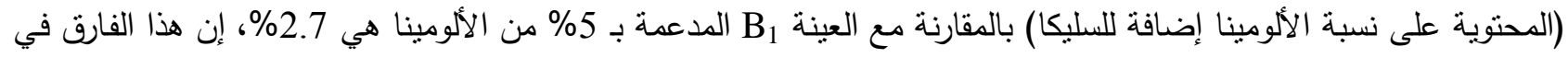

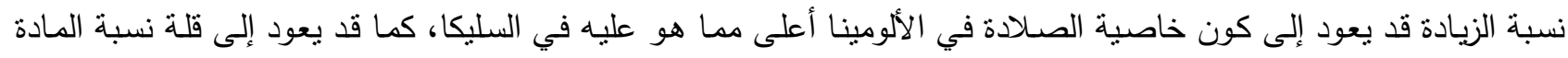

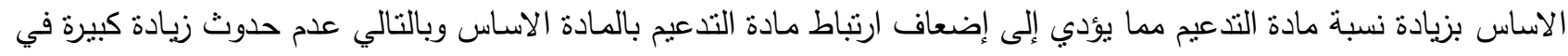
قيم الصلادة بزيادة التدعيم. هذه النتائج تتقق مع ما نوصل إليه (Garay et al., 2011)، و (Abbas et al., 2015).

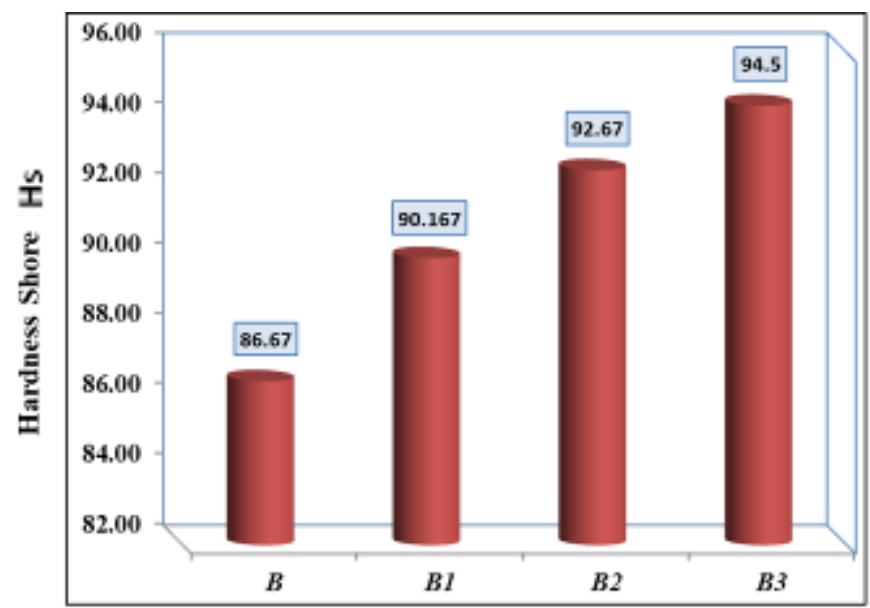




\section{Impact Test إختبار الصدمة}

يبين اختبار الصدمة مدى مقاومة المادة للانهيار عند تعرضها لقوى صدم تحت ظروف التشغيل، إذ إن معدل امتصاص الطاقة عند الصدم يؤثز على سلوك المواد وبالتالي على جودتها (المؤسسة العامة للتنريب المهني والتقني، 2008)، يبين الثكل (8) مقاومة الصدمة للعينات المحضرة. ومن خلال نتائج الإختبار نجد أن مقاومة الصدمة للمواد المتراكبة قد انخفضت عند التذعيم عما هو عليه الحال في المادة الاساس B ولجميع نسب التدعيم. حيث إن المواد المتراكبة تتكون من ثلاث مناطق رئيسية

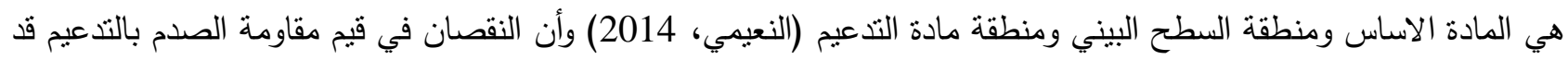

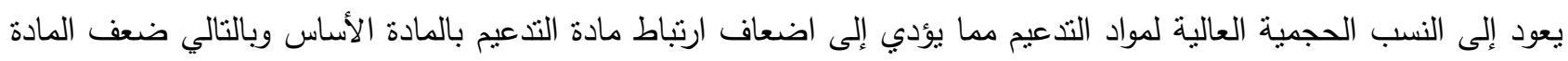

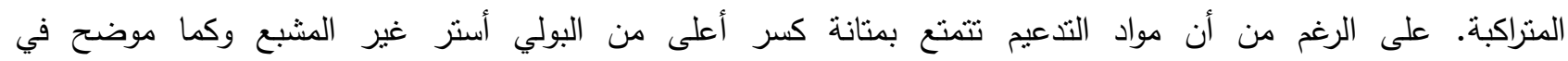

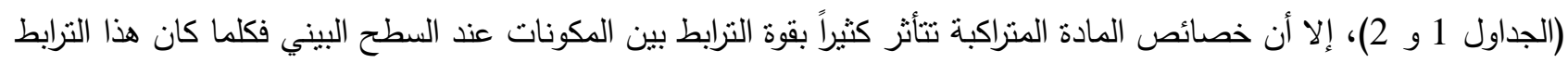
قوياً أدى ذلك إلى زيادة تأثز المادة الاساس بخصائص مواد التدعيم. إذ تنتقل الإجهادات المسلطة على المادة المتراكبة عبر المادة

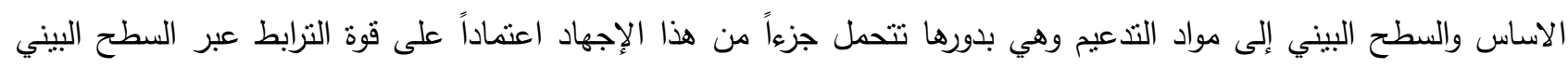

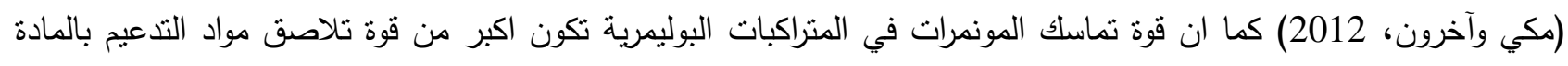

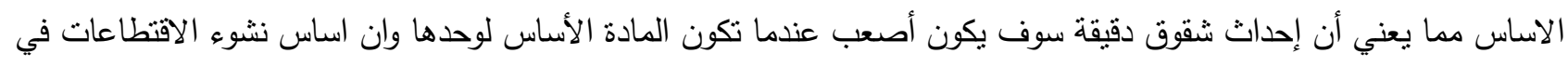

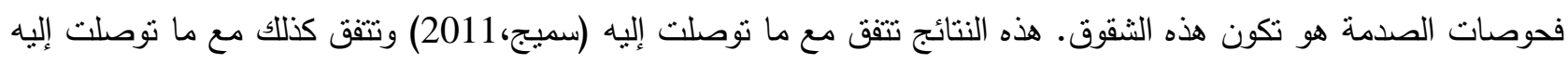
حيث انخفضت مقاومة الصدمة بالتدعيم غير إنها ازدادت زيادة طفيفة بعد نسبة التدعيم Abbas et al., 2015)

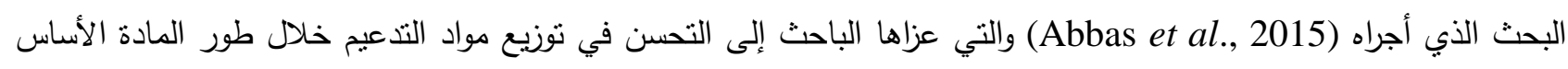

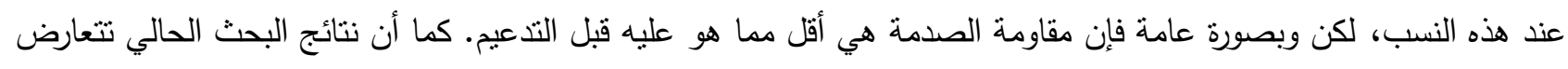
مع ما نوصلت إلبه (Abas and Abass, 2018) والتي استخدمت نسب تدعيم من مسحوق كاربيد السليكون ضمن الددى هي 1\% vol\%

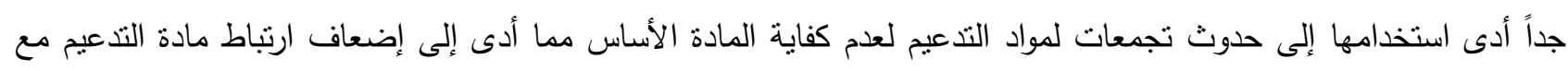
المادة الأساس وتجمع مواد التدعيم مع بعضها في مناطق محدودة وبالتالي تحسين تكون الفجوات وبالنتيجة يؤدي إلى تكون

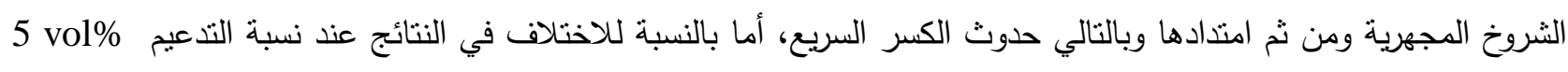
فإنه يعود ذللك إلى الفرق في حجم دقائق التدعيم التي استخدمها (Abas and Abass, 2018) والتي تراوحت ما بين Mm (60-25) وهي صغيرة مقارنة مع المدى الحجمي لمواد التدعيم المستخدمة في البحث الحالي مما أدى إلى إسهام هذه فئه

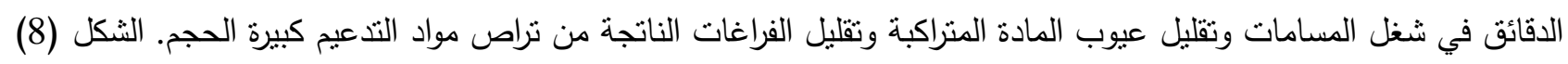

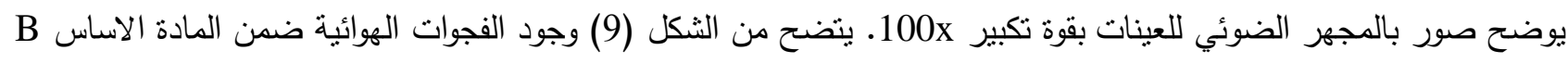
بسبب الخلط عند القولبة اليدوية كما يلاحظ زيادة المسامات بزيادة التدعيم والذي يضعف من بوفئين الخصائص الميكانيكية للمواد

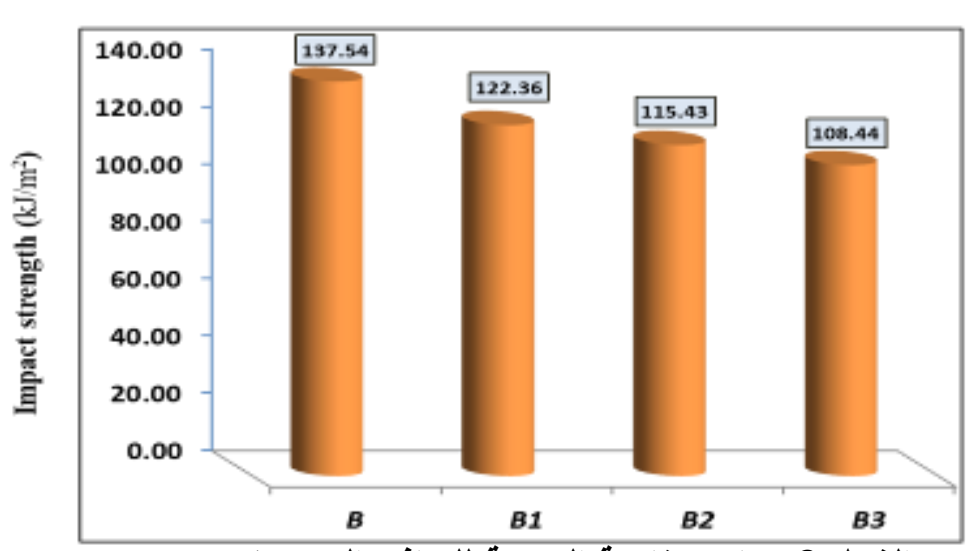

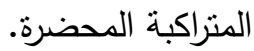

الثكل 8: نتائج مقاومة الصدمة للنماذج المحضرة. 

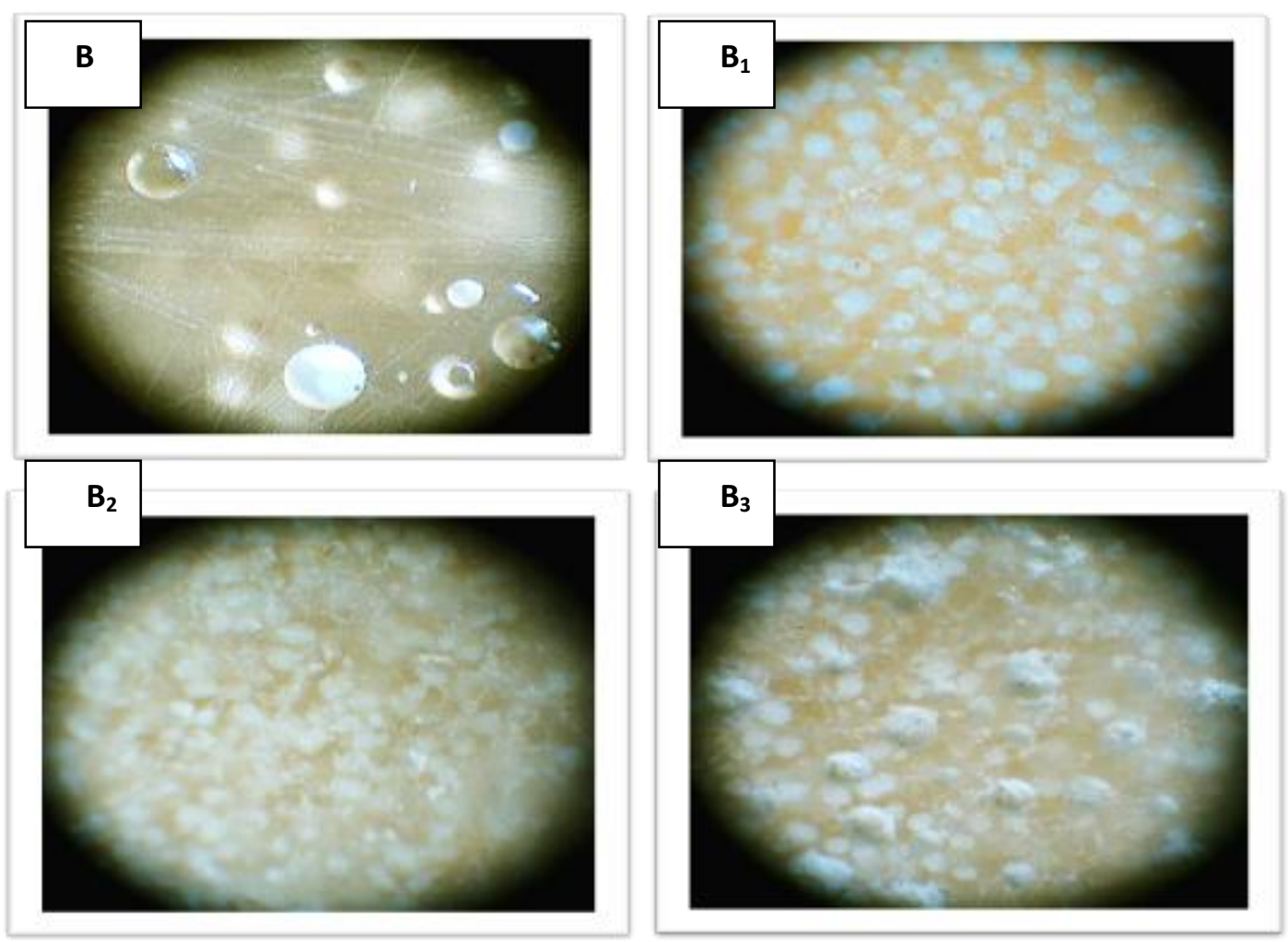

الشكل 9: صور مجهرية بقوة 100x للنماذج المحضرة B, B 1 , B

الاستنتاجات

من خلال هذه الدراسة تم التوصل للاستتتاجات التالية:

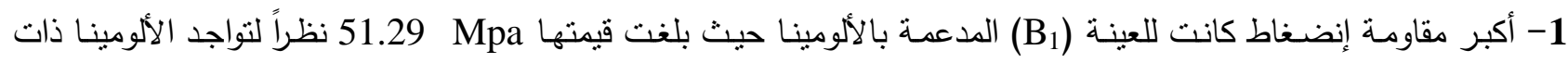

مقاومة الإنضغاط العالية مقارنة بالمادة الاساس (B). 2- زيادة الصـلادة بزيادة التدعيم وقد بلغت أقصـاها (B خصائصها مع خصائص مواد التدعيم.

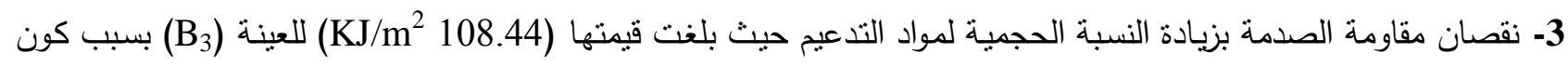
ترابط المونمرات في المادة الاساس اقوى من الترابط بين المادة الاساس ومواد التدعيم، حيث تقل نسبة المادة الأساس بزيادة نسبة التدعيم. 4- نقصان مقاومة الكسر في إختبار الانحناء بزيادة نسبة مواد التدعيم بسبب نقصان نسبة المادة الأساس بزيادة نسبة التدعيم مما يؤدي الى ضعف الارتباط بين المادة الأساس ومواد التدعيم ونقصان في خصائص اللدونة اللّة المستمدة من المادة الاساس.

\section{المصادر العربية}

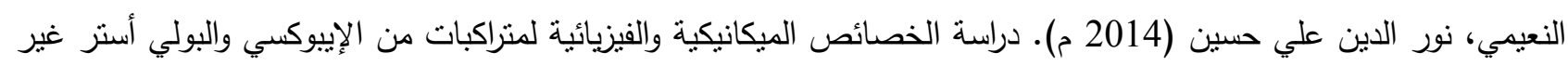
المشبع المقواة بألياف الزجاج ومسحوق الألموينا النانوي والمايكروي. رسالة ماجستير ، جامعة الموصل. العزاوي، علي حسن رسن هذال؛ الدباغ، بلقيس محمد ضياء؛ الحداد، سلام حسين علي (2010). تأثثر درجة الحرارة والمحاليل الكيميائية على قيم معامل المرونة لمواد منراكبة هجينة. مجلة الهندسة والتكنلوجيا. 22 (13) ، 693-708. 
الرفاعي، جمال؛ السويلم، فارس. (2011). "البوليمرات"، مدينة الملك عبد العزيز للعلوم والتقنية. معهد بحوث البتروكيمياويات. المملكة العربية السعودية.

المؤسسة العامة للتدريب التقني والمهني، الإدارة العامة لتصميم وتطوير المناهج، (2008). "إختبار المواد". المملكة العربية السعودية.

تاكر ، آنا أ، ترجمة محمد، أكرم عزيز، (1984). "الكيمياء الفيزيائية للبوليمرات". طبع بمطابع جامعة الموصل. حسن، أحمد غازي (2017). دراسة تأثنر إضافة دقائق السليكا على الخواص الميكانيكية لمتراكبات البولي أستر غير المشبع

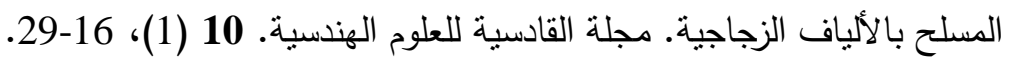

حسين، مظفر يعقوب؛ حسين، رحيم جعفر (2010). تحسين الخواص الميكانيكية والحرارية للبولي يوريثان. مجلة كلية التربية

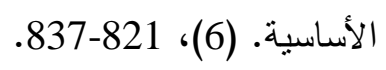

سميج، هناء عرير (2011). تأثثر التقوية بمسحوق أوكسبد الزنك على الخواص الميكانيكية لمادة متراكبة ذات أساس من البولي

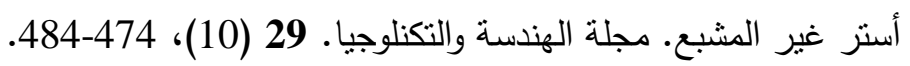

عاصي، عبد الله ضايع؛ عبود، أحمد هادي؛ جواد، زهراء فخري. (2015). دراسة الخواص الميكانيكية لمادة البولي أستر غير المشبع مقوى بمسحوق التيتانيوم والألومينا. مجلة جامعة بابل/ العلوم الهندية، رهراء. 23 (1)، 52-62.

مرهون، إسماعيل إبراهيم؛ صلال، حيدر عباس (2014). دراسة تأثير إضافة دقائق بابل مennie الصبدلانية على مواد متراكبة ذات

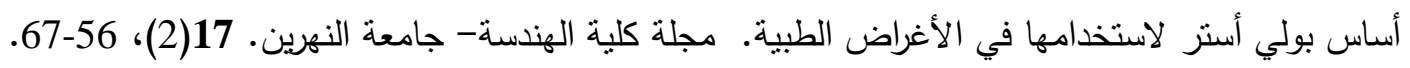

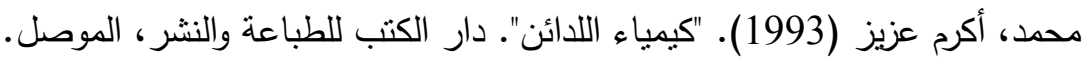

مكي، سمير عطا؛ أحمدز عدي حميد؛ عبد الله، مصطفى زيد؛ غني، بسمة علي (2012). دراسة تأثير التدعيم ودرجة الحرارة في

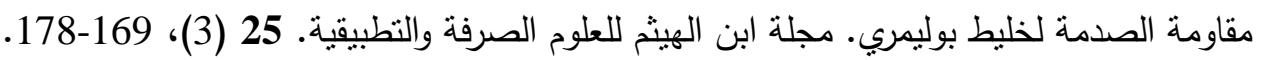

\section{المصادر الأجنبية}

Abas, F.O.; Abass, R.U. (2018). Study thermo-mechanical properties of polyester composite reinforced by ceramic particles Sic. MATEC Web of Conf., 225 (01021).

Abbas, A.F.; Betti, N.A.; Abbas, R.U. (2015). Study themomechanical properties of unsaturated polymer composite reinforced by ceramic particles (Al2O3). IJISET-Int. J. Inn. Sci., 2, $150-152$.

Akay, M. (2012). "Introduction to Polymer Science and Technology". Mustafa Akay and Ventus Publishing APS.

Battelle, (2008). "Material Science and Technology". Handbook, National Technical Information Service, U.S Department of Energy, 5285 Port Royal Rd., http://www.ntis.gov/ordering.htm, email: orders@ @its.fedworld.gov.

Callister, W. D. (2009). "Materials Science and Engineering". John Wiley and Sons, Inc., $8^{\text {th }}$ ed.

Coble, S. (2003). "Materials Data Book". Cambridge University Engineering Department, Cambridge, UK.

Doremus, R.H.; Shackelford, J. F. (2008). "Ceramic and Glass Materials Structure, Properties and Processing". Springer.

Durowaye, S.; Sekunowo, O.; Shittu, S.; Duru, P. (2018). Effect of silicon carbide, millscale, and magnesia particulates on the mechanical properties of hybrid unsaturated polyester resin matrix composites. Usak Uni. J. Eng. Sci., 1 (1), 28-37.

Ebewele, R.O. (2000). "Polymer Science and Technology". CRC Press LLC, Nigeria. 
Garay, A.C.; Heck, V.; Zattera, A.J.; Souza, J.A.; Amico, S.C. (2011). Influence of calcium carbonate on $\mathrm{rtm}$ and $\mathrm{rtm}$ light processing and properties of molded composite. J. Rei. Pla. and Com., 30 (14), 1213-1221.

Hanna, W.A.; Gharib, F. E.; Marhoon, I.I. (2011). Characterization of ceramic filled polymer matrix composite used for biomedical applications. J. Min. and Mat. Char. Eng., 10 (12), 1167-1178.

Hyer, M.W. (2009). "Stress Analysis of Fiber-Reinforced Composite Materials". DE Stech Publications Inc.

Kim, J.H.; Kim, S.C. (1987). Analysis of reaction injection molding process of polyurethaneunsaturated polyester blends. part II: mechanical properties. Pol. Eng. Sci., 27(16), 12521257.

Majeed, A.H.; Ibrahim, S.Q. (2017). Mechanical properties of unsaturated polyester filled with silica fume, glass powder and carbon black. Eng. Tech. J., 35 (6), 640-647.

Musa, B. H. (2014). Effect of glass powder on some mechanical properties of polymer matrix composite material. J. Al-Nah. Uni., 17 (2), 108-115.

Crawford, R. J. (1998). "Plastics Engineering". $3^{\text {rd }}$ ed., printed by St Edmunds bury press Ltd, Bury St Edmunds, Suffolk.

Ratna, D. (2009). "Handbook of Thermost Resins". Smithers Rapra Tech.

Rodriguez, G.P.; Maldonado, LM.; Moreno, H.J.D. (2015). Structure, mechanical and thermal behavior of mixtures of polyester resin and dental ceramic waste. J. Phy., 687 (012086).

\title{
Reinforcement Effect of Alumina and Silica on the Mechanical Properties of Mixture Polyesters (Unsaturated Polyester / Polyurethane)
}

\author{
Haneen M. Ali Raad A. Rasool Soham Y. Moustafa \\ Department of Physics/ College of Education for Pure Science / University of Mosul
}

\begin{abstract}
In this study composite materials were prepared from based polymer mixture (B) (UPE/PU, 95/5) supported by alumina powders and silica with grain sizes $(75-150) \mu \mathrm{m}$, these samples were tested $\mathrm{B}$ and $\mathrm{B}_{1}, \mathrm{~B}_{2}$ and $\mathrm{B}_{3}$ respectively.

The samples were subjected to mechanical tests represented by bending test, compression, hardness and impact at laboratory temperature. The results showed that the Young modulus of bending test increased by increasing the strength, and the fracture resistance was reduced by increasing the reinforcement. As for compressive resistance, the maximum compressive resistance was for alumina - supported $\left(\mathrm{B}_{1}\right)$ sample $51.29 \mathrm{MPa}$. The Hardness was increased by increasing reinforcement with a maximum value of $94.5 \mathrm{Hs}$.
\end{abstract}

Keywords: Composite materials, Polymer Mixture, Mechanical Properties. 